\title{
The Emergent Universe: An explicit construction
}

\author{
George F.R. Ellis, Jeff Murugan and Christos G. Tsagas \\ Department of Mathematics and Applied Mathematics, \\ University of Cape Town, Rondebosch 7700, Cape Town, South Africa
}

(Dated: May 30, 2018)

\begin{abstract}
We provide a realization of a singularity-free inflationary universe in the form of a simple cosmological model dominated at early times by a single minimally coupled scalar field with a physically based potential. The universe starts asymptotically from an initial Einstein static state, which may be large enough to avoid the quantum gravity regime. It enters an expanding phase that leads to inflation followed by reheating and a standard hot Big Bang evolution. We discuss the basic characteristics of this Emergent model and show that none is at odds with current observations.
\end{abstract}

\section{INTRODUCTION}

The idea that the universe we inhabit might be in a state of eternal inflation is not new. In fact it was realized quite soon after its initial proposal that inflation is usually future eternal; that is, in most cases there will always be regions of spacetime that are inflating in the future. So a natural question arises as to whether the universe was always inflating in the past; could inflation be past eternal also? Recent work by several authors 1] seems to indicate that the answer to this intriguing question is decidedly "no"! Using Penrose-HawkingGeroch techniques, it is argued in detail that a spacetime that $(i)$ is past causally simple, $(i i)$ is open, $(i i i)$ is described by Einstein's equations with a matter source that obeys the weak energy condition, and (iv) allows for inflation to be future eternal, cannot be past null complete. Crucial to the argument is the assumption that the universe is open or at least flat. As is often pointed out though, we are emerging into an era of "precision cosmology" and measurements of temperature anisotropies in the Microwave Background are able to place greater constraints on the curvature of the universe than ever before 2]. The recent WMAP data seems to point to a universe that is close to (but not quite) flat, with a total density parameter of $\Omega_{t o t}=1.02 \pm 0.02$. Nevertheless, until we have something like a three sigma signal, it remains premature to conclude with any certainty whether we live in a flat universe or not 34. In particular, the WMAP data does not rule out closed models. In this light, it would seem that we have a promising avenue around the arguments of [1]. If we live in a universe that is closed (albeit only marginally so) today, then it was always closed, and perhaps inflation is past-eternal after all.

Recently arguments were put forward for several inflationary cosmologies that were past-eternal while avoiding any quantum gravity regime [3]. Each of the proposed models is spatially closed and described only with general relativity, ordinary matter, and minimally coupled scalar fields. Their existence argues against the suggestions of 1] that inflationary universes are necessarily bounded in the past, and shows that a quantum gravity dominated era for the universe may not be inevitable; something that has also been noted in [4]. The EddingtonLemaitre cosmology is a well-known example of a universe that is not past geodesic-incomplete, because of its positively curved spatial sections. Harrison has also given an exact solution with similar properties [5]. His is a radiation-dominated closed universe with a positive cosmological constant. It starts from an Einstein static state, with a radius determined by the value of $\Lambda$, before entering a never-ending period of de Sitter expansion. However both these models do not exit inflation. Here, following [3] , we consider a universe filled with a dynamical scalar field, which is past asymptotic to an Einstein static model with a radius determined by the field's kinetic energy. This model enters a period of de Sitter inflation that comes naturally to an end as the scalar field starts oscillating around the minimum of the potential, before entering the standard hot Big-Bang expansion phase. Thus these are singularity-free inflationary universe models 35], by-passing the restrictions of the singularity theorems mentioned above [36]. They are finely tuned in terms of the initial conditions, although one can use entropy arguments to favor an initial Einstein Static phase for our universe 27]. We consider possible implications of this fine-tuning in the Conclusion.

\section{A POTENTIAL EMERGENT POTENTIAL}

Slightly more than a decade ago it was shown 7] that by an inversion of the conventional viewpoint (beginning with a scalar field whose self interaction is dictated by some underlying particle physics considerations and subsequently determining the evolution of the universe) scalar field dynamics could be explicitly accounted for without a slow-roll approximation. Indeed a scalar field potential $V(\phi)$ could quite easily be 'reverse engineered' for almost any desired behavior of the scale factor $a(t)$. Several examples were explicitly computed and are summarized: 


\begin{tabular}{|c||c||}
\hline$a(t)$ & $V(\phi)$ \\
\hline$A \exp (\omega t)$ & $3 \kappa^{-1} \omega^{2}+\omega^{2}\left(\phi-\phi_{0}\right)^{2}$ \\
$A \sinh (\omega t)$ & $3 \kappa^{-1} \omega^{2}+B^{2} \sinh ^{2}\left(2 \omega\left(\phi-\phi_{0}\right) / B\right)$ \\
$A \cosh (\omega t)$ & $3 \kappa^{-1} \omega^{2}+B^{2} \sin ^{2}\left(2 \omega\left(\phi-\phi_{0}\right) / B\right)$ \\
$A t^{n}$ & $(3 n-1) B^{2} \exp \left( \pm 2\left(\phi-\phi_{0}\right) / B\right) / 2$ \\
\hline
\end{tabular}

where $\kappa=8 \pi G$. The above respectively correspond to de Sitter exponential expansion, de Sitter expansion from a singularity, de Sitter expansion without a singularity, and power-law expansion respectively [7]. The eternal emergent universe, a nonsingular model past asymptotic to an Einstein static universe with topology $\mathbf{R} \times S^{3}$ and radius $a_{0} \gg L_{P l}$, can be treated in this way too. For a prescribed scale factor behavior of $a(t) \sim a_{0}+\exp (h t)$ an associated potential is not too difficult to compute (see the appendix for details). While it certainly produces the desired early time behavior, the potential does not have a definitely zero minimum. This is an undesirable feature as it points to a rather large cosmological constant, although there are ways around it. Also, because of the limits on the integration in the reverseengineering construction, a graceful exit from the inflationary regime is not always guaranteed. Consequently we need to find a universe with a potential that is essentially the same as that in the eternal emergent universe at very early times but then goes to zero at some finite value of the field. That is, we need to look for a potential $V(\phi)$ that matches onto the reconstructed potential (Fig. 6) as $t \rightarrow-\infty$, with a long flat plateau, but then has a vanishing minimum value.

Fortunately we need not look too far. Inflationary models based on higher derivative curvature terms go back to the remarkably prescient work of Starobinsky [8] in which the de Sitter phase was driven by the trace anomaly of the energy momentum tensor (see also [9]). Among the variants on the original Starobinsky model, $R^{2}$-inflation based on a Lagrangian of the form $\mathcal{L}=$ $R+\alpha R^{2}$ exhibits a particularly elegant implementation of a de Sitter phase with a linearly decaying Hubble parameter, $H$. The $R^{2}$ term in this action is effectively an additional scalar degree of freedom which may be absorbed by the introduction of a (non-dynamical) scalar field [10].37] Einstein gravity is restored by an appropriate conformal transformation but at the expense of a dynamical scalar field with an interesting potential 11, 13, 14, 15]. Since we build our model from a related potential, it is worth seeing how this works. Starting from an $R^{2}$-modified action

$$
\begin{aligned}
S & =\int \mathrm{d}^{4} x \sqrt{-g}\left[R+\alpha R^{2}\right] \\
& =\int \mathrm{d}^{4} x \sqrt{-g}\left[(1+2 \alpha R) R-\alpha R^{2}\right],
\end{aligned}
$$

define $\Omega^{2}:=1+2 \alpha R$ and make the conformal transformation $g_{\mu \nu} \mapsto \widetilde{g}_{\mu \nu}=\Omega^{2} g_{\mu \nu}$ so that $\sqrt{-\widetilde{g}}=\Omega^{4} \sqrt{-g}$ and

$$
\widetilde{R}=\frac{1}{\Omega^{2}}\left[R-6 g^{\mu \nu} \nabla_{\mu} \nabla_{\nu}(\ln \Omega)-6 g^{\mu \nu} \nabla_{\mu}(\ln \Omega) \nabla_{\nu}(\ln \Omega)\right]
$$

Substituting this into (1) gives

$$
\begin{aligned}
S & =\int \mathrm{d}^{4} x \sqrt{-\widetilde{g}}\left[\widetilde{R}-\frac{\alpha}{\Omega^{4}} R^{2}\right] \\
& +6 \int \mathrm{d}^{4} x \sqrt{-g} \Omega \nabla_{\mu}\left(g^{\mu \nu} \partial_{\nu} \Omega\right)
\end{aligned}
$$

The last term may be evaluated by noting that the divergence $\nabla_{\mu} X^{\mu}=(1 / \sqrt{-g}) \partial_{\mu}\left(\sqrt{-g} X^{\mu}\right)$. With this,

$\int \mathrm{d}^{4} x \sqrt{-g} \Omega \nabla_{\mu}\left(g^{\mu \nu} \partial_{\nu} \Omega\right)=-\int \mathrm{d}^{4} x \sqrt{-\widetilde{g}} \frac{1}{\Omega^{2}} \widetilde{g}^{\mu \nu} \partial_{\mu} \Omega \partial_{\nu} \Omega$

after a boundary term is discarded. The action for the $R^{2}$ model in the Einstein frame is then written as

$$
\begin{aligned}
S & =\int \mathrm{d}^{4} x \sqrt{-\widetilde{g}}\left\{\widetilde{R}-\frac{6 \alpha^{2}}{(1+2 \alpha R)^{2}}\left[\widetilde{g}^{\mu \nu} \partial_{\mu} R \partial_{\nu} R+\right.\right. \\
& \left.\left.+\frac{1}{6 \alpha} R^{2}\right]\right\}
\end{aligned}
$$

To make contact with a canonical form for scalar field actions [15], it is usual to rewrite the scalar degree of freedom offered by the scalar curvature as $\varphi:=\sqrt{3} \ln (1+$ $2 \alpha R)$ so that

$$
S=\int \mathrm{d}^{4} x \sqrt{-\widetilde{g}}\left\{\widetilde{R}-\frac{1}{2} \widetilde{g}^{\mu \nu} \partial_{\mu} \varphi \partial_{\nu} \varphi-\frac{1}{4 \alpha}\left(e^{-\varphi / \sqrt{3}}-1\right)^{2}\right\} .
$$

The effective potential in (4) is just the reflection of that in Fig. [1 about $\phi=0$. Note that the parameters describing the potential are fairly rigidly constrained (with respect to $\alpha$ ) by the conformal transformation [38].

Since it's conception the $R^{2}$ model has received a significant amount of attention, largely as a result of the fact that the scalar field driving the de Sitter phase arises so naturally and is not inserted "by hand" solely to provide the inflationary dynamics. Indeed it was shown in 13. that the model quite naturally supports a transient period of inflation followed by a FRW universe. Constraints on the coupling constant $\alpha$ are imposed by requiring that density perturbations be of an appropriate magnitude. Consequently, $10^{12} M_{P l}^{-2} \lesssim \alpha \lesssim 10^{16} M_{P l}^{-2}$. This in turn determines the height of the plateau of the potential to be of the order $10^{-13}-10^{-17} \times M_{P l}^{4}[16]$. This form of the potential will form the cornerstone of our construction of the Emergent universe.

\section{DETERMINATION OF THE PARAMETERS IN THE POTENTIAL}

Emergent type universes can be realized by relaxing the constraints on the $R^{2}$ effective potential and considering a spacetime filled with a minimally coupled, single 


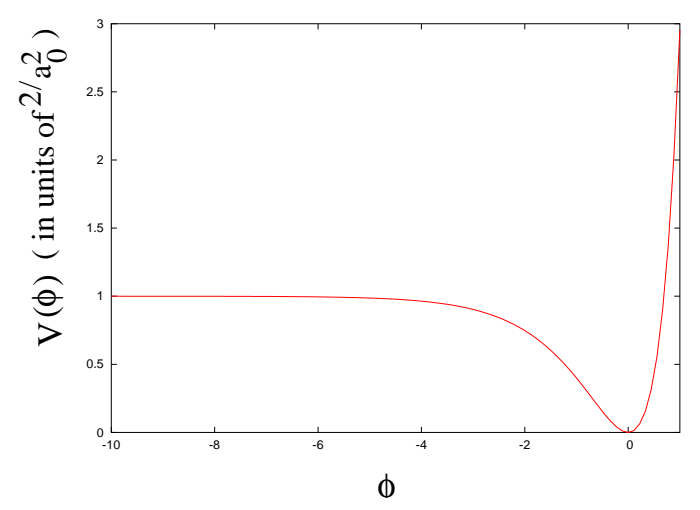

FIG. 1: The Emergent potential for the parameter choice $B=1$ is identical up to an overall rescaling to the effective potential for the scalar field in the Einstein frame of the $R^{2}$ driven inflation model after relabeling $\phi$ as $-\varphi$.

scalar field $\phi$ with a potential of the general form

$$
V=V(\phi)=\left(A \mathrm{e}^{B \phi}-C\right)^{2}+D
$$

where $A, B, C$ and $D$ are constants to be determined by the specific properties of the emergent universe. Then,

$$
V^{\prime}(\phi)=2 A B\left(A \mathrm{e}^{B \phi}-C\right) \mathrm{e}^{B \phi},
$$

and

$$
V^{\prime \prime}(\phi)=2 A B^{2}\left(2 A \mathrm{e}^{B \phi}-C\right) \mathrm{e}^{B \phi},
$$

where a prime indicates a derivative with respect to $\phi$. Therefore, the above potential has a minimum at $\phi_{0}=$ $(1 / B) \ln (C / A)$ with $V_{0}=V\left(\phi=\phi_{0}\right)=D$. Consequently, if we want to set the minimum of the potential at the origin of the axes, we must choose $A=C$ and $D=0$. Note that zero minimum for $V(\phi)$ guarantees that there is no residual cosmological constant. Then, expression (5) reduces to

$$
V(\phi)=A\left(\mathrm{e}^{B \phi}-1\right)^{2} .
$$

By definition, the emergent universe corresponds to a past-asymptotic Einstein-static (ES) model [3]. This means that

$$
V(\phi \rightarrow-\infty)=\frac{2}{\kappa a_{0}^{2}},
$$

where $a_{0}$ is the radius of the initial static model 28]. It is then clear that for $a_{0} \gg L_{P l}$, where $L_{P l}$ is the Planck length, the model can avoid the quantum regime. To determine an additional parameter recall that an ES universe filled with a single scalar field satisfies the condition $V(\phi)=2 / \kappa a_{0}^{2}=\dot{\phi}$, where $\kappa=8 \pi G$ [28]. Hence, for an initially ES state we require that $A=2 / \kappa a_{0}^{2}$, which brings the expression of the potential down to

$$
V(\phi)=\frac{2}{\kappa a_{0}^{2}}\left(\mathrm{e}^{B \phi}-1\right)^{2} .
$$

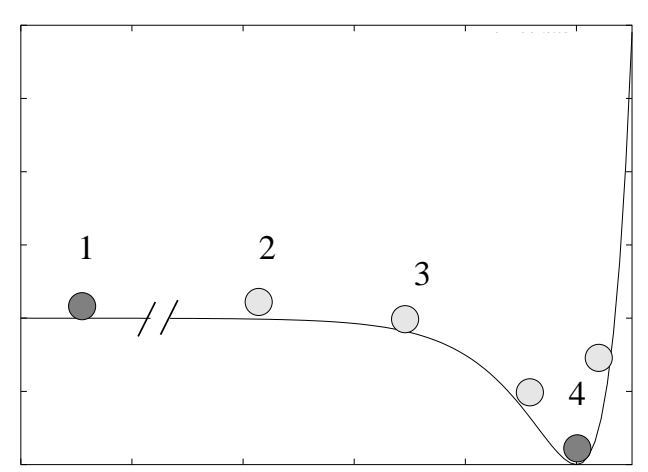

FIG. 2: A schematic illustration of the scalar field evolution in the Emergent universe. After leaving its initial static state (1) the model enters a slow-rolling regime (see case (ia)), or it goes through an intermediate pre-slow-roll phase (see case(ib)) (2). In either case the scale factor grows sufficiently quickly to mitigate neglecting the curvature effects. A period of slowroll (3) inflation is followed by a re-heating phase (4)and then by the standard hot Big Bang evolution.

Therefore, the basic properties of the emergent universe have already fixed three of the four parameters in the original potential given by Eq. (5). The remaining parameter $(B)$ will be determined by considering other aspects of the model and in particular by looking into the density perturbation spectrum (see Sec. VIII below). Nevertheless, we can still determine, the sign of $B$ by demanding, without loss of generality, that

$$
V^{\prime}=\frac{4 B}{\kappa a_{0}^{2}}\left(\mathrm{e}^{B \phi}-1\right) \mathrm{e}^{B \phi}<0
$$

for $-\infty<\phi<0$. This means that $\mathrm{e}^{B \phi}-1<0$ and consequently that $B>0$. Incidentally, on comparison with the corresponding potential of the $R^{2}$ action, this is seen to correspond to a choice of negative coupling constant $\alpha$ as required to avoid manifesting tachyons and singular perturbative behavior in the model [15].

\section{LEAVING THE EINSTEIN-STATIC REGIME}

As the universe leaves the ES state the evolution of the scalar field is determined by the Klein-Gordon equation

$$
\ddot{\phi}+3 H \dot{\phi}+V^{\prime}=0,
$$

where $H=\dot{a} / a$ is the Hubble parameter. Therefore, given that $\dot{\phi}>0$, we have expansion only if $\ddot{\phi}<-V^{\prime}$. Since the potential drops with increasing $\phi$, we may consider the following two alternative cases:

(i) $\ddot{\phi}<0$, namely a decelerated scalar field, where the friction for the deceleration comes from the expansion. In this case one expects the kinetic energy of $\phi$ drops. 
Then, since $\dot{\phi}^{2} \simeq V$ initially and $V^{\prime} \simeq 0$, a slow-rolling period with $\dot{\phi}^{2} \ll V$ seems likely.

(ii) $0 \leq \ddot{\phi}<-V^{\prime}$, that is a "slowly" accelerating scalar field. Then, one expects the kinetic energy of the scalar field to increase. Under these conditions, a slow-rolling regime seems unlikely. Therefore, we are left with case (i), which splits further into two subcases:

(ia) $V^{\prime}<\ddot{\phi}<0$, namely a "weakly" decelerated scalar field, monitored by the familiar expression associated with slow-roll inflation

$$
3 H \dot{\phi}+V^{\prime}=0
$$

since $|\ddot{\phi}|<\left|V^{\prime}\right|$; and

(ib) $\ddot{\phi}<V^{\prime}<0$, that is a "strongly" decelerated scalar field, described by the following form of the Klein-Gordon equation

$$
\ddot{\phi}+3 H \dot{\phi}=0
$$

since $|\ddot{\phi}|>\left|V^{\prime}\right|$.

So, as the universe leaves the ES state, it may evolve in a number of ways depending on the relation between $\ddot{\phi}$ and $V^{\prime}$. Cases (i) and (ii) lead to expanding models, of which (i) is the alternative corresponding to the Emergent Universe. Of the two possible subcases of (i), the first leads immediately to the standard slow-rolling inflationary regime. In the next sections we will concentrate primarily on this particular case. Before we proceed further, however, we should make the following comment with regard to case (ib). It involves a strongly decelerated, that is a very slow-rolling scalar field. Nevertheless, Eq. (14) also implies that $\dot{\phi} \propto a^{-3}$. The latter suggests that, as $\phi$ drops rapidly, subcase (ib) could also lead to the familiar slow-rolling inflation. We may test this possibility by considering the reverse engineered potential given in the Appendix (see Eqs. (A13), A14), where $\ddot{\phi}$ dominates over $V^{\prime}$ just like in (ib). Then, the point where the two potentials deviate should determine the initial conditions for the Einstein field equations that describe subcase (ib).

\section{THE DURATION OF THE SLOW-ROLL REGIME}

We solve numerically and plot the results for the scale factor in Fig. 3] The graph clearly shows that $a$ has the familiar exponential increase associated with standard slow-rolling inflationary models while at earlier times approaches a constant non-zero value.

The duration of the slow-rolling inflationary regime, in a spatially flat model, is determined by the usual slow-

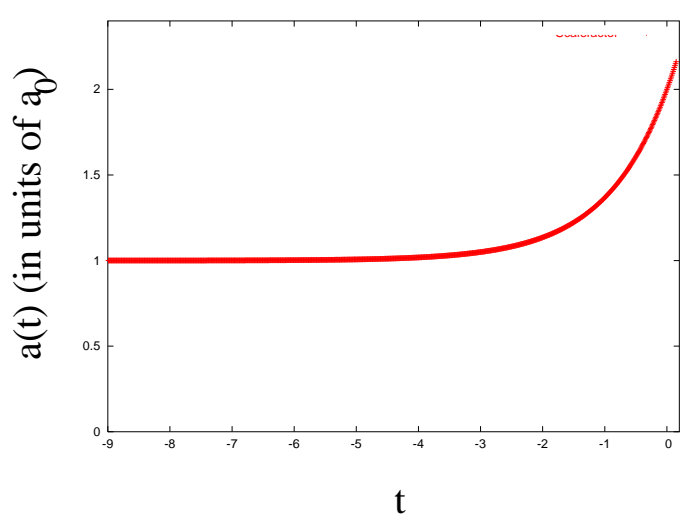

FIG. 3: The initial evolution of the scale factor in the Emergent universe.

roll parameters 17

$$
\epsilon(\phi)=\frac{1}{2} M_{P l}^{2}\left(\frac{V^{\prime}}{V}\right)^{2} \quad \text { and } \quad \eta(\phi)=M_{P l}^{2} \frac{V^{\prime \prime}}{V},
$$

where $M_{P l}$ is the Planck mass. Throughout slow-roll approximation, the above satisfy the constraints $\epsilon(\phi) \ll$ 1 and $|\eta(\phi)| \ll 1$, which provide the limits of the slow-roll regime. Applied to the potential of the emergent universe (given by Eq. (10), with $B>0$ and for $-\infty<\phi<0$ ), the above constraints read

$$
\epsilon(\phi)=\frac{2 B^{2} \mathrm{e}^{2 B \phi}}{\left(\mathrm{e}^{B \phi}-1\right)^{2}} \ll 1
$$

and

$$
|\eta(\phi)|=\left|\frac{2 B^{2}\left(2 \mathrm{e}^{B \phi}-1\right) \mathrm{e}^{B \phi}}{\left(\mathrm{e}^{B \phi}-1\right)^{2}}\right| \ll 1,
$$

respectively, where we have set $M_{P l}=1$ for simplicity. It should be emphasised that, although our model is spatially closed, the effect of the curvature (which is dominant at very early times) becomes negligible after few e-foldings, and only re-emerges in the recent universe.

After a rather lengthy, but fairly straightforward analysis, one can show that neither constraint provides a lower bound for $\phi$. Therefore, the slow-roll regime starts at an arbitrarily small value of $\phi$. In practice, this means that the emergent model starts slow rolling at a few efoldings after leaving its initial ES state, at a finite $\phi_{i}$, when the curvature effects have become negligible. Given that the static regime corresponds to $\phi \rightarrow-\infty, \phi_{i}$ is very small and the available number of e-foldings can be very large.

Note that, by employing (16) and (17), we can also show that for any positive value of $B>0$ there is always a negative value for $\phi$ at which the slow-rolling regime ends i.e., where $\epsilon, \eta \simeq 1$. For example, when $B=1$ we find that $\phi<-\ln (1+\sqrt{2})$ (for $M_{P l}=1$ ). 


\section{THE NUMBER OF E-FOLDINGS}

For standard slow-roll inflation the number of efoldings is given by the expression [18]

$$
N\left(\phi_{i} \rightarrow \phi_{f}\right)=\int_{t_{i}}^{t_{f}} H \mathrm{~d} t
$$

where $\phi_{i}, \phi_{f}$ and $t_{i}, t_{f}$ are the initial and final values of $\phi$ and $t$ respectively, and $H$ is the Hubble parameter. The latter is generally given by 3,28$]$

$$
H^{2}=\frac{1}{3} \kappa\left(\frac{1}{2} \dot{\phi}+V\right)-\frac{k}{a^{2}},
$$

where $k=0, \pm 1$ is the 3 -curvature index. After few efoldings, however, the effect of the curvature term becomes negligible, while for slow-roll inflation we have $\dot{\phi}^{2} \ll V$. Thus, throughout the slow-rolling regime $H=\sqrt{\kappa V / 3}$. Combining expression (18) with this result we obtain

$$
N=-\kappa \int_{\phi_{i}}^{\phi_{f}} \frac{V}{V^{\prime}} \mathrm{d} \phi
$$

given that $\mathrm{d} t=\mathrm{d} \phi / \dot{\phi}$ and that $\dot{\phi}=-V^{\prime} / \sqrt{3 \kappa V}$, as the slow-rolling version of the Klein-Gordon equation (i.e. with $\ddot{\phi} \simeq 0$ ) guarantees.

Applying the above to the case of the emergent universe, namely inserting the potential (10), we arrive at the expression

$$
N=-\frac{\kappa}{2 B} \int_{\phi_{i}}^{\phi_{f}} \frac{\mathrm{e}^{B \phi}-1}{\mathrm{e}^{B \phi}},
$$

which provides the number of e-foldings associated with the emergent universe as a function of the yet undetermined parameter $B$. This is easily computed numerically, and in Fig. 4 we plot $N$ against the value of $\phi$ at the start of the slowroll period for various values of the parameter $B$. Clearly, depending on the value of $\phi_{i}$, sufficient e-folds are easily obtainable in this model. Note that $N$, while certainly very large, is nevertheless finite since the value of $\phi_{i}$ can be stretched back only to the point where the curvature effects become appreciable. From a different point of view, the reason there are only a finite number of efoldings is because the initial value of the scale-factor is non-zero (unlike the standard inflationary model with $K=0)$.

\section{SCALE FACTOR EVOLUTION}

Starting from the Friedmann equation of the slowrolling inflationary regime (see Eq. (19)) we have

$$
\frac{\dot{\phi}}{a} \frac{\mathrm{d} a}{\mathrm{~d} \phi}=\sqrt{\frac{1}{3} \kappa V} .
$$

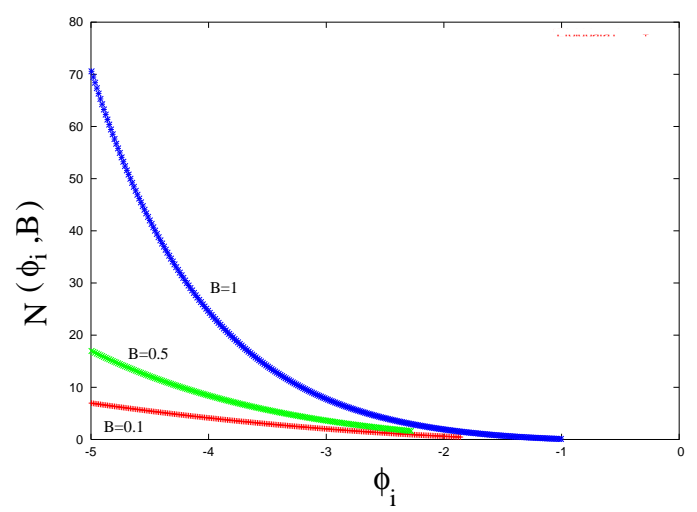

FIG. 4: The number of e-folds obtained during a slowroll regime in the Emergent model plotted against $\phi_{i}$ and for varoius values of the parameter $B$.

At the same time, the Klein-Gordon equation gives $\dot{\phi}=$ $-V^{\prime} / \sqrt{3 \kappa V}$, having set $\ddot{\phi} \simeq 0$. Combining the two we arrive at

$$
\frac{1}{a} \mathrm{~d} a=-\frac{\kappa V}{V^{\prime}} \mathrm{d} \phi
$$

Applied to the emergent universe, by using expressions (10) and (11), the above lead to the differential equation

$$
\frac{1}{a} \mathrm{~d} a=\frac{\kappa}{2 B} \frac{1-\mathrm{e}^{B \phi}}{\mathrm{e}^{B \phi}} \mathrm{d} \phi,
$$

which integrated gives

$$
\ln \left(\frac{a}{a_{i}}\right)=-\frac{\kappa}{2 B^{2}}\left(\mathrm{e}^{-B \phi}-\mathrm{e}^{-B \phi_{i}}\right)-\frac{\kappa}{2 B}\left(\phi-\phi_{i}\right) .
$$

So, the value of the scale factor at the end of the slowrolling regime depends crucially on $\phi_{i}$, namely on the value of $\phi$ at the onset of slow-roll inflation. As expected, the smaller $\phi_{i}$ is the larger the final value of $a$.

\section{THE DENSITY SPECTRUM}

For structure formation purposes it is crucial to determine the density contrast at horizon crossing 50 efoldings before the end of inflation. Following [18] we have

$$
\left(\frac{\delta \rho}{\rho}\right)_{H o r} \simeq\left(\frac{H^{2}}{\dot{\phi}}\right)_{N=50} \simeq-\left(\frac{3 H^{3}}{V^{\prime}}\right)_{N=50} .
$$

During the slow-rolling regime the Hubble parameter is given by Eq. (19) and the above takes the form

$$
\left(\frac{\delta \rho}{\rho}\right)_{H o r} \simeq-\sqrt{\frac{1}{3} \kappa^{3}}\left(\frac{V^{3 / 2}}{V^{\prime}}\right)_{N=50} .
$$


Substituting the potential of the emergent universe (see Eq. (10)), we obtain the following expression for the density contrast associated with the model

$$
\left(\frac{\delta \rho}{\rho}\right)_{H o r} \simeq \frac{\kappa}{\sqrt{6} B a_{0}}\left[\frac{\left(\mathrm{e}^{B \phi}-1\right)^{2}}{\mathrm{e}^{B \phi}}\right]_{N=50},
$$

which also depends on the yet undetermined parameter $B$. In view of the COBE observations the above is constraint by

$$
\frac{\kappa}{\sqrt{6} B a_{0}}\left[\frac{\left(\mathrm{e}^{B \phi}-1\right)^{2}}{\mathrm{e}^{B \phi}}\right]_{N=50} \simeq 10^{-5} .
$$

Consequently, the CMB anisotropy limits allow us to express $B$ as a function of $a_{0}$, the radius of the initial ES state, which therefore becomes the key parameter of the emergent model. In Fig. 5 we plot $\delta \rho / \rho$ against $a_{0}$ for several values of the parameter $B$. The appropriate value of the Einstein radius is then read off from the intersection of the perturbation curves with $\delta \rho / \rho=10^{-5}$. For example, the $B=1$ curve satisfies the density perturbation requirement when $a_{0} \simeq 10^{6} L_{P l}$. Again a comparison with the corresponding $R^{2}$ potential shows that $\alpha \sim a_{0}^{2} \sim 10^{12} M_{P l}^{-2}$, within the required range for that parameter [16]. This result fixes the remaining parameter in our initial potential (see Eq. (5) leaving us with just one more free parameter; the scalar field value when slow-roll commences $\phi_{i}$.

Having used the amplitude of fluctuations to constrain the parameters of the model, it remains to check that other quantities whose values are tightly constrained by CMB data remain within their experimental bounds. Among these we count the power and polarization spectra and the spectral index of the density fluctuations. As a preliminary check, we note that the spectral index of scalar perturbations may quite easily be estimated by following the prescription of [20]. We thus define the horizon-flow parameters $\epsilon_{1}$ and $\epsilon_{2}$ through the usual slowroll parameters $\epsilon$ and $\eta$ as

$$
\begin{aligned}
& \epsilon_{1}:=\epsilon / 8 \pi \\
& \epsilon_{2}:=(\epsilon-2 \eta) / 4 \pi
\end{aligned}
$$

in terms of which the spectral index $n_{S}-1=$ $d \ln \mathcal{P}_{k} / d \ln k=-2 \epsilon_{1}-\epsilon_{2}$. Using the expressions (16) and (17) for the slowroll parameters, an estimate for the first horizon-flow parameter is obtained as $\epsilon_{1} \sim 0.00014$. Subsequent computation of the spectral index yields $\left|n_{S}-1\right| \sim 0.012$ or $n_{S} \sim 0.987$. This result, although only a rough estimate is certainly compatible with those of [2, 21]. We expect that a computation of the power spectrum will not deviate significantly from the standard result either and hope to report on this in forthcoming work [22].

With the above value for the Einstein static radius, we anticipate that the CMB anisotropy spectrum will have

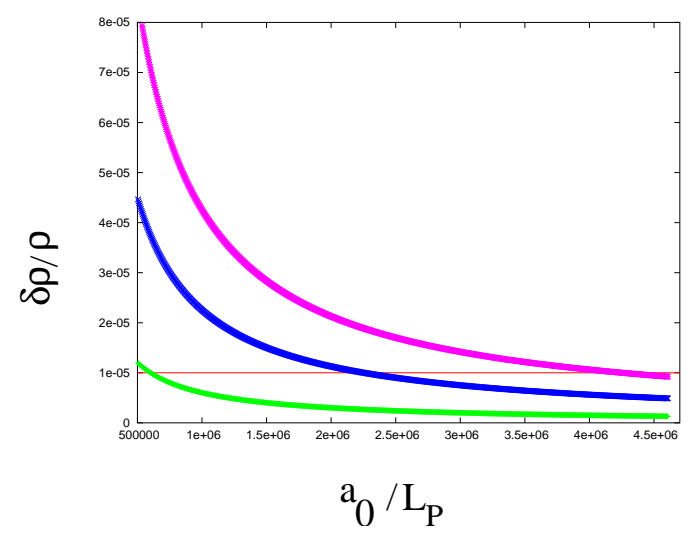

FIG. 5: Plots of $\delta \rho / \rho$ against the size of the Einstein static universe $a_{0}$ for various values of the parameter $B$. As $B$ increases from $B=0.1$ through $B=1$ the value of $a_{0}$ for which $\delta \rho / \rho \sim 10^{-5}$ increases from $a_{0} \sim 6.02 \times 10^{5} L_{P l}$ to $a_{0} \sim 0.42 \times 10^{7} L_{P l}$

the usual Sachs-Wolfe plateau and peaks as confirmed by current observations [39]. Moreover, once the scalar field potential is fixed we have a residual one-parameter family of models - parameterized by $\phi_{i}$ - all asymptotic to the same Einstein static universe and within which is contained a class of models with $\Omega_{t o t}=1+\varepsilon$ where $\varepsilon$ is arbitrarily small. Since these models are all closed and begin from the same state they serve well to illustrate the emergent model. However, the fact that $\Omega_{t o t}$ is not forced to be 1.02 avoids both excessive fine-tuning of the Einstein static radius and a possible cosmic coincidence problem [40].

\section{AVAILABLE ENERGY FOR REHEATING}

Inflation starts at $V(\phi \rightarrow-\infty)=2 / \kappa a_{0}^{2}$ and ends at $\phi=0$ with $V_{0}=0$, which means that the maximum energy "stored" in the inflaton field is

$$
V(\phi \rightarrow \infty)=\frac{2}{\kappa a_{0}^{2}} \simeq \frac{10^{37} \mathrm{GeV}^{2}}{a_{0}^{2}} .
$$

In other words, the maximum energy available for reheating is inversely proportional to the square of the radius of the initial ES state. If $a_{0}=10^{6} L_{P l}$ we find

$$
V(\phi \rightarrow \infty) \simeq 10^{63} \mathrm{GeV}^{4} .
$$

Therefore, provided that thermal equilibrium has been achieved and that the reheating process is efficient, the temperature at the beginning of the standard Big Bang evolution can be as high as

$$
T_{R H} \simeq 10^{16} \mathrm{GeV} .
$$

Obviously, for less efficient reheating $T_{R H}<10^{16} \mathrm{GeV}$. 


\section{DISCUSSION}

Closed inflationary models have not had a happy time of late. Indeed many authors have taken the latest measurements of Microwave Background isotropies, in particular the WMAP best fit estimate of $\Omega_{t o t}=1.02 \pm 0.02$ to signal a flat infinite universe. Yet there are two ways to interpret these observations. It could be that the universe is in fact flat and more precise experiments in the future will eventually whittle away at the error bars until $\Omega_{t o t}=1$ to within, say, one part in a million. On the other hand, a comparison of BOOMERANG data 23] (with a best fit of $\Omega_{t o t}=1.02_{-0.05}^{+0.06}$ ) with WMAP data would seem to indicate a convergence on $\Omega_{t o t}=1.02>1$ with increasing resolution. Admittedly, the data set from which we draw this conclusion is rather limited and it is with much eagerness that we await results from the European Space Agency's PLANCK satellite. However, until this debate is settled one way or the other, one is forced to take closed models seriously. In stark contrast to some claims in recent literature [24], it is not too difficult to construct consistent, single field inflationary models in a closed universe. We give one such construction here (but invite the reader to see 25] for another, simple yet remarkably elegant model).

The "Emergent Universe" proposed in [3] is a simple closed inflationary model in which the universe emerges from an Einstein static state with radius $a_{0} \gg L_{P l}$, inflates and is then subsumed into a hot Big Bang era. The attractiveness of the proposed model is that one can avoid an initial quantum-gravity stage if the Einstein Static radius is larger than the Planck length. One might then ask whether such a model has a simple representation and whether it lies within the boundaries of current observations. In this paper, we provide a first explicit construction of such a universe. As such, it is a manifestly nonsingular closed inflationary cosmology that begins from a meta-stable Einstein static state and decays into a de Sitter phase and subsequently into standard hot Big Bang evolution. Inspired by an exact reverse-engineered potential constructed as in 7] (see the appendix), this phenomenological model employs a single scalar field with a potential very similar to that arising in conformally transformed $R^{2}$-inflation only with a relaxing of some of the rigidity of that potential. In particular, beginning with a four-parameter potential (5), we can immediately fix two of the parameters by fixing the origin of the potential and requiring vanishing cosmological constant. By requiring this potential to match onto the exact reverse-engineered one at early times $(|\phi| \gg 1)$, a third parameter of the potential is related to the radius of the initial Einstein universe by $A^{2}=2 / \kappa a_{0}^{2}$. The remaining two-parameter model is then shown to exhibit all the desired properties of the Emergent universe model. Among others, these include a sufficient number of e-folds to solve the late-time flatness problem, a spectrum of density fluctuations with magnitude of the order of $10^{-5}$ and sufficient energy in the scalar field to allow for adequate post-inflationary reheating. Of these we find that the spectrum of density fluctuations provides another constraint that allows us to relate the remaining two parameters so that effectively, the only parameter in the model is the size of the Einstein static universe. As for the re-heating energy, we show that for an initial radius of $a_{0}=10^{6} L_{P l}$ we can obtain re-heating temperatures of around $10^{16} \mathrm{GeV}$.

Of course, an immediate question arises as to a viable mechanism that realizes the initial Einstein static universe that our model emerges from. At this point several avenues present themselves. Among the more promising, we note two; the first of these is the observation of 29] that the Einstein static universe is one of only two asymptotic solutions of the Ramond-Ramond sector superstring cosmology field equations [41]. The size of the positively curved Einstein universe in this picture is controlled by the level number of the Kac-Moody algebra of the conformal fields living in the compactified internal space. Indeed, this observation leads quite naturally to the tantalizing possibility of realizing an Emergent-like universe within a string cosmology context [30]. A second, equally intriguing, possibility is that the initial Einstein static universe is created from "nothing" by some quantum tunneling process [31, 32]. Indeed, finiteness of the tunneling action requires that the universe created through instanonic tunneling be closed [33]. It is not implausible, then, that through spontaneous quantum fluctuations, a closed universe could be created in a long lived but transient Einstein static state which then makes a transition to a finite lifetime de-Sitter and subsequent marginally closed FRW phase along the lines described above. It remains to be seen that such a mechanism can be concretely realized in any quantum cosmological framework.

The attractive aspects of the Einstein Static solution as a preferred initial state for our universe have been considered in the past. In fact, Gibbons has argued for the higher probability of an Einstein Static initial phase based on the model's maximal entropy [27]. Crucially, once the universe finds itself near the static state it could remain there for an undetermined amount of time. This is guaranteed by the neutral stability of the Einstein Static model against inhomogeneous (either pure fluid or pure scalar field) perturbations [26, 27, 28]. Typically, expansion away from the static solution will lead to inflation followed by the standard Hot Big Bang evolution. However, the reader will probably by now have noted the large degree of fine-tuning that went into setting up the initial state from which the universe emerges. Indeed, the emergent model is a very special trajectory in the space of possible inflationary evolutions. We have shown existence of such models, but not that they are probable.

Some may regard this as a deadly blow to these models, but we believe the case is wide open. Firstly, we note that although the idea that the universe should be prob- 
able (and so not fine-tuned) is the dominant paradigm in cosmology at present, there is no scientific proof that this has to be the case. This is an unproven and indeed unprovable philosophical assumption, which may or may not be true [42]. It is equally conceivable that - as was taken for granted in the past - whatever process causes the universe to come into being prefers a high-symmetry state. It is then relevant that the Einstein-static model is the highest symmetry non-empty Robertson-Walker universe, and so would be preferred by such a process.

Secondly, the models presented here show one can avoid the initial singularity if initial conditions were fine tuned in the way remarked on above. We believe it likely that this is a generic result: that given the usual physics of inflationary fields in the early universe (i.e. avoiding the introduction of 'shadow matter' which violates the weak energy condition), there is either a singularity at the start of the universe [43] or a fine-tuned initial state. This may be the real philosophical choice facing us: to decide which is worse, a space-time singularity, with all that that entails, or a fine tuning of initial conditions. It certainly seems very difficult to (phenomenologically, at least) construct a model that avoids both, and it is useful to recall Wheeler's characterization of space time singularities caused by gravitational collapse as the worst crisis facing theoretical physics. Nowadays we do not perhaps take such singularities seriously enough.

Models of the kind presented here are useful in terms of making clear the alternatives facing us: we can indeed avoid both a singularity and the quantum gravity regime, without introducing any exotic physics; but there is a price to pay in terms of fine-tuning. From some philosophical standpoints the high symmetry of the initial state may even be an advantage.

\section{APPENDIX A: AN EXACT SOLUTION}

Consider a Friedmann-Robertson-Walker (FRW) universe containing a minimally coupled scalar field $\phi$ with Lagrangian density

$$
\mathcal{L}=\frac{1}{2}(\partial \phi)^{2}-V(\phi)=\frac{1}{2} \dot{\phi}^{2}-V(\phi)
$$

where the last equality follows from assuming spatial homogeneity of the $\phi$ field i.e. $\phi=\phi(t)$. With this assumption, the stress-energy tensor takes the form of a perfect fluid with energy density and pressure

$$
\begin{aligned}
& \rho_{\phi}=\frac{1}{2} \dot{\phi}^{2}+V(\phi) \\
& p_{\phi}=\frac{1}{2} \dot{\phi}^{2}-V(\phi)
\end{aligned}
$$

respectively [4]]. The classical equation of motion for $\phi$ that follows from variation of the action $S=\int \mathrm{d}^{4} x \sqrt{-g} \mathcal{L}$ is

$$
\ddot{\phi}+3 H \dot{\phi}+\frac{d V(\phi)}{d \phi}=0
$$

where the Hubble parameter $H:=\dot{a}(t) / a(t)$. The Raychaudhuri field equation for the FRW model with scalar field matter source and its first integral, the Friedmann equation are

$$
3 \dot{H}+3 H^{2}=8 \pi G\left(V(\phi)-\dot{\phi}^{2}\right)
$$

and

$$
3 H^{2}+3 \frac{k}{a^{2}}=8 \pi G\left(\frac{1}{2} \dot{\phi}^{2}+V(\phi)\right)
$$

respectively. These equations, together with (12) for a closed dynamical system from which the evolution of the universe model is determined. However, it is important to note that the Klein-Gordon equation is auxiliary in the sense that any solution of (A4) and $\mathrm{A} 5$ with nonvanishing $\dot{\phi}$ will necessarily satisfy (12) so that the dynamical system in fact contains only two independent equations [45]. These may be combined 7] to give the (more convenient) equivalent set of equations

$$
\begin{aligned}
V(\phi(t)) & =\frac{1}{8 \pi G}\left(\dot{H}+3 H^{2}+2 \frac{k}{a^{2}}\right) \\
\dot{\phi}^{2}(t) & =\frac{1}{4 \pi G}\left(\frac{k}{a^{2}}-\dot{H}\right) .
\end{aligned}
$$

With these equations at hand, the potential $V(\phi)$ is contructed by

- Specifying the constant $k$ and a particular (monotonic) function $a(t)$ and computing the associated Hubble parameter $H$ and $\dot{H}$.

- Checking that the constraint $k / a^{2}-\dot{H} \geq 0$ is met. This assures the positivity of $\dot{\phi}^{2}$ as is necessary for a neutral scalar field.

- Specifying an initial condition $\phi_{0}$ for $\phi(t)$ and integrating the second of eqs. A6 to get $\phi(t)$. This is then inverted (where possible) to give $t(\phi)$.

- Substituting into the first of eqs. A6 to obtain $V(t)=V(t(\phi))$ and subsequently $V(\phi)$.

Thus, as advertised, this algorithm associates to a specified scale factor $a(t)$ a potential $V(\phi)$ to give a model that exactly solves the classical inflationary field equations. At this point some comments are in order: First, the reconstruction of the potential is manifestly non-unique as should be clear from the sign ambiguity in the choice of root in the $\dot{\phi}$ equation (A6). Secondly, it is not clear how sensitive this algorithm is to the choice of initial conditions for $\phi$ i.e. on where on the potential the scalar field resides when it begins to roll. While general shape should be independent it might be expected that the details of the potential (location of extrema etc.) would vary with different initial conditions. This, however, does not affect the argument. 


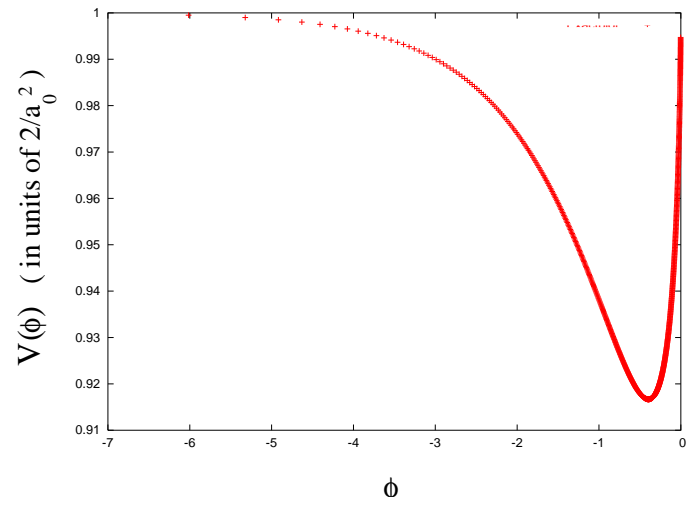

FIG. 6: A parametric plot of the re-constructed potential corresponding to the exact solution $a(t)=a_{0}+\exp (h t)$ normalized with respect to the size of the Einstein static universe. This re-constructed potential exhibits the same shape as the effective potential of the $R^{2}$ model in the Einstein frame but is plagued by a rather shallow minimum (only about $91 \%$ of its asymptotic value.)

The emergent model is essentially a modified version of the Eddington-Lemaitre universe - $k=+1$, pastasymptotically Einstein static, singularity-free, without particle horizons and ever-inflating (see [3] for more details). Before applying the potential reconstruction technique to this model, a few general observations about the scenario beg attention. The Einstein static state containing matter with energy density $\rho_{i}$ and pressure $p_{i}=w_{i} \rho_{i}$ $\left(-1 / 3<w_{i}<1\right)$ is characterized by

$$
\begin{aligned}
\frac{1}{2}\left(1-w_{i}\right) \rho_{i}+V(\phi) & =\frac{1}{4 \pi G a_{0}^{2}}, \\
\left(1+w_{i}\right) \rho_{i}+\dot{\phi}_{i}^{2} & =\frac{1}{4 \pi G},
\end{aligned}
$$

where $a_{0}$ is the radius of the $S^{3}$ spatial sections of the universe. If the sole content of this universe is the scalar field, as is the case in this note, $\rho_{i}=0$ and the second of eq. A77) requires that the scalar field have non-vanishing (but constant) kinetic energy. In this model with no matter initially present, the mechanism envisaged in [3] has $\phi$ rolling at a constant speed along a flat potential $\left(V=V_{i}\right)$ from $\phi=-\infty$ at $t=-\infty$ to $\phi=0$ at $t=0$ where the potential first rises (and inflation is initiated) and then drops to a minimum at $\phi=\phi_{f}$ where the value of the potential is $V_{f}=\Lambda / 8 \pi G \ll V_{i}$. The field is carried over the hill by its non-zero kinetic energy and slow rolls toward the minimum where its damped oscillations reheats the universe. What follows is an attempt to realise this scenario.

To this end, and to facilitate numerical computations it will prove useful to rescale quantities of interest as

$$
\begin{aligned}
V(\phi) \rightarrow M_{P l}^{4} V(\phi), & \phi(t) \rightarrow M_{P l} \phi(t), \\
t \rightarrow t / M_{P l}, & a(t) \rightarrow a(t) / M_{P l},
\end{aligned}
$$

where $M_{P l}^{2}:=8 \pi G$. In these units the Raychaudhuri equation and its first integral, the Friedmann equation become

$$
\begin{aligned}
3 \dot{H}+3 H^{2} & =V(\phi)-\dot{\phi}^{2}, \\
3 H^{2}+3 \frac{1}{a^{2}} & =\frac{1}{2} \dot{\phi}^{2}+V(\phi),
\end{aligned}
$$

which, together with the Klein-Gordon equation form the dynamical system governing the evolution of the closed scalar field dominated FRW universe. The corresponding equations A6 for the potential and $\dot{\phi}(t)$ that are the starting point for the reconstruction of the potential take the form

$$
\begin{aligned}
V(\phi(t)) & =\dot{H}+3 H^{2}+\frac{2}{a^{2}} \\
\dot{\phi}^{2}(t) & =2\left(\frac{1}{a^{2}}-\dot{H}\right) .
\end{aligned}
$$

Following [3] consider the scale factor

$$
a(t)=A+B \exp (h t),
$$

where $A, B, h$ are all positive constants. This universe is past asymptotic to an Einstein static phase, since $a(t) \rightarrow$ $A$ as $t \rightarrow-\infty$. Thus, $A$ is identified with the radius $a_{0}$ of the Einstein static universe. At late times, on the other hand, $a(t) \rightarrow B \exp (h t)$ and the model approaches a de Sitter expansion phase. The second of eqs. (A10) then gives

$$
\phi(b)=\sqrt{2} \int \frac{d b}{h b} \sqrt{\frac{1-h^{2} a_{0} b}{\left(a_{0}+b\right)^{2}}},
$$

where $b:=B \exp (h t)$. The integral is easily evaluated to give

$$
\begin{aligned}
\phi(b)= & \frac{\sqrt{2}}{h a_{0}} \ln \left[\left(\frac{\sqrt{1+h^{2} a_{0}^{2}}+\sqrt{1-h^{2} a_{0} b}}{\sqrt{1+h^{2} a_{0}^{2}}-\sqrt{1-h^{2} a_{0} b}}\right)^{\sqrt{1+h^{2} a_{0}^{2}}} .\right. \\
& \left.\cdot\left(\frac{1-\sqrt{1-h^{2} a_{0} b}}{1+\sqrt{1-h^{2} a_{0} b}}\right)\right],
\end{aligned}
$$

and with the $b$ parameterization the potential is written

$$
V(b)=\frac{3(h b)^{2}+h^{2} a_{0} b+2}{\left(a_{0}+b\right)^{2}} .
$$

At this stage, an expression for $V(\phi)$ is, in principle, obtained by inverting eq. (A13) and substituting $b(\phi)$ into eq. A14 . However the above form of $\phi(b)$ precludes such a simple treatment. Nevertheless, the general shape of $V(\phi)$ is easily determined by a parametric plot. This is given in Fig [ 6

\section{ACKNOWLEDGMENTS}

G.F.R.E thanks the NRF(South Africa) for funding this work, J.M. is supported by a research associateship of the University of Cape Town and the Lindbury trust and C.T. acknowledges financial support from 
a Sida/NRF fund and a DAA bursary. We thank Bill Stoeger for very helpful contributions and have enjoyed very fruitful discussions with John Barrow, Peter
Dunsby, Roy Maartens, Jean-Philippe Uzan and Amanda Weltman at various stages of this work.
[1] A. Borde and A. Vilenkin, Phys. Rev. Lett. 723305 (1994); ibid, Phys. Rev. D 56, 717 (1997); A. Borde, A.H. Guth and A. Vilenkin (astro-ph/0110012); A.H. Guth, (astro-ph/0101507); A. Vilenkin, (astro-ph/0204507)

[2] D.N. Spergel et. al., First year Wilkinson anisotropy probe (WMAP) observations: Determination of Cosmological Parameters, ApJ, To appear (2003)

[3] G.F.R Ellis and R. Maartens, The Emergent Universe: Inflationary cosmology with no singularity and no quantum gravity era, gr-qc/0211082

[4] C. Molina-Paris and M. Visser, Phys. Lett. B 455, 90 (1999)

[5] E.R. Harrison, Mon. Not. R. Astron. Soc. 137, 69 (1967)

[6] G.F.R. Ellis, Closed Trapped Surfaces in Cosmology, gr-qc/0304039

[7] G.F.R. Ellis and M.S. Madsen, Exact scalar field cosmologies, Class. Quantum Grav. 8, 667 (1991)

[8] A.A. Starobinskii, A new type of isotropic cosmological model without singularity, Phys. Lett.91B, 99 (1980)

[9] S.W. Hawking et. al. Trace anomaly driven inflation, Phys. Rev. D 63, 083504 (2001)

[10] D. Wands Extended gravity theories and the EinsteinHilbert action, Class. Quantum Gravity 11, 269 (1994)

[11] B. Whitt, Fourth-order gravity as general relativity plus matter, Phys. Lett.145B, 176 (1984)

[12] E.I. Guendelman, Scale invariance, Inflation and Lambda terms, Mod. Phys. Lett. A14, 1043-1052 (1999), gr-qc/9901017

[13] K. Maeda, Inflation as a transient attractor in $R^{2}$ cosmology, Phys. Rev. D 37, 858 (1988)

[14] S. Kalara et. al. Theories of inflation and conformal transformations, Nucl. Phys. B 341, 252 (1990)

[15] J. Barrow The premature recollapse problem in closed inflationary universes, Nucl. Phys. B 296, 697 (1988)

[16] M.B. Mijic, M.S. Morris and W.-M. Suen, The $R^{2}$ cosmology: Inflation without a phase transition, Phys. Rev. D 34, 2934 (1986)

[17] A.R. Liddle and D.H. Lyth: Cosmological Inflation and Large-Scale Structure (Cambridge University Press, Cambridge 2000)

[18] E.W. Kolb and M.S. Turner, The Early Universe (Addison-Wesley, 1990)

[19] J.-P. Luminet, J. R. Weekes, A. Riazuelo, and R. Lehoucq. To appear (2003).

[20] S.M.Leach, A.R. Liddle, J. Martin and D.J. Schwartz, Cosmological parameter estimation and the inflationary cosmology, astro-ph/0202094

[21] A.M. Leach and A.R. Liddle, Constraining slow-roll inflation with WMAP and 2dF, astro-ph/00306305

[22] G.F.R. Ellis, J. Murugan and C. G. Tsagas, In Progress

[23] P. de Bernardis et. al. Multiple Peaks in the Angular Power Spectrum of the Cosmic Microwave Background: Significance and Consequences for Cosmology, Astrophys. J. 564 559-566 (2002)

[24] A. Linde, Can we have inflation with $\Omega>1$, astro-ph/0303245
[25] A. Lasenby and C. Doran, Closed Universes, de Sitter Space and Inflation, astro-ph/0307311

[26] E.R. Harrison. Rev Mod Phys 39, 862 (1967).

[27] G.W. Gibbons, Nucl. Phys. B 292, 784 (1987); ibid, 310, 636 (1980)

[28] J.D. Barrow, G.F.R. Ellis, R. Maartens and C.G. Tsagas, Class. Quantum Grav. 20, L155 (2003)

[29] I. Antoniadis, C. Bachas, J. Ellis and D.V. Nanopolous, Phys. Lett. B 211, 4 (1988)

[30] J. Murugan, G.F.R. Ellis and A. Weltman, In progress

[31] E.P. Tryon, Nature(London) 246, 396 (1973)

[32] A. Vilenkin, Phys. Rev. D 32, 10 (1985)

[33] S. Gratton, A. Lewis and N. Turok, Closed Universes from Cosmological Instantons,Phys. Rev. D 65, 043513 (2002), astro-ph/0111012

[34] We thank the referee for reminding us of this point.

[35] One objection that could be raised at this point is the persistence of closed trapped surfaces in this cosmological model and the consequent implications for the existence of a cosmological singularity. Indeed such past closed trapped surfaces do exist but as shown in [6] do not imply the existence of a cosmological singularity in the model.

[36] The assumptions of the singularity theorems were later relaxed [1] and it was pointed out that essentially the same argument holds for any spacetime in which null geodesics do not recross after traversing the entire universe. This is not true for the Einstein static spacetime so the models presented here safely fall out of the domain of the Borde-Vilenkin theorem.

[37] This scalar field is in fact an auxiliary one whose 'equation of motion' may be trivially solved to show that it is nothing but the scalar curvature in disguise.

[38] In fact, such a potential is not unique to higher order gravity theories. In particular, it has been shown that such potentials appear quite naturally in models of spontaneously broken scale invariance [12].

[39] Indeed the following observation is relevant: the model proposed here can have the standard $S^{3}$ topology for the positively curved spatial surfaces, but that is not obligatory; they could for example be Poincaré dodecahedral spaces with positive spatial curvature, as suggested by Luminet et al [19]. In that case they might give a better fit to the WMAP data at large angular scales than other models [19].

[40] We thank the referee for pointing this out.

[41] The other being a linearly expanding Milne universe

[42] The proposal of an actually existing multiverse, which could possibly be used to justify the idea that the universe must indeed be probable, is also unprovable.

[43] or at least non-avoidance of the quantum gravity domain, when quantum gravity processes may possibly avoid the singularity.

[44] More generally, the FRW universe may also be assumed to contain some noninteracting perfect fluid with energy density $\rho$ and pressure $p=w \rho$ with $-\frac{1}{3}<w \leq 1$. The 
inclusion of such additional matter sources is however unnecessary for the purpose of this work.

[45] If $\dot{\phi}$ does indeed vanish then the Klein-Gordon equation is no longer a consequence of the other two equations but may nevertheless be easily solved to yield a constant potential $V(\phi)=$ const. 